\title{
A Novel Interference Avoidance Technique on Mobile Wireless Routers Using IEEE802.11n PSMP
}

\author{
Akira KISHIDA $^{\dagger}$, Takefumi HIRAGURI ${ }^{\dagger \dagger}$, Masakatsu OGAWA ${ }^{\dagger \dagger}$, Members, \\ Kentaro NISHIMORI ${ }^{\dagger+\dagger}$, Senior Member, Naoki HONMA ${ }^{\dagger+\dagger \dagger}$, and Tetsu SAKATA ${ }^{\dagger}$, Members
}

\begin{abstract}
SUMMARY This paper proposes an interference avoidance technique that allows wireless device with similar frequency bands to be operated adjacent to each other for compact mobile wireless routers (MWRs). This MWR implements two devices of Wireless LAN (WLAN) and Worldwide Interoperability for Microwave Access (WiMAX). The MWR connects WLAN terminals to the backbone network by using WiMAX-WLAN relay. Generally, different frequency channels are assigned for the wireless systems assign in order not to interfere among multiple systems. However, mutual system interference is generated if the space between each device is very close and if the frequency using each system is adjacent. To suppress this interference, this paper proposes a novel interference avoidance technique that leverages IEEE802.11n Power Save Multi-Poll (PSMP). First, we clarify the conditions that raise the issues of mutual interference by experiment. Simulations are conducted to show that the proposed scheme outperforms the conventional schemes. Finally, the effectiveness of the proposed scheme is shown by the computer simulation.

key words: IEEE802.11n, IEEE802.16e, mutual system interference, PSMP, wireless relay
\end{abstract}

\section{Introduction}

Recently, wireless LANs that comply with IEEE802.11 standard [1] are widely implemented in various mobile terminals not only laptop PCs but also small multimedia devices such as music players, gaming machines, and smart phones. Demand has been growing that these mobile terminals be usable everywhere. However, WLAN service areas are restricted to WLAN hotspots in public areas. In order to solve this problem, mobile wireless routers (MWRs), which can connect WLAN terminals to the backbone network by using other wireless system with wide communication area, are a hot research topic [2]-[4]. On the other hand, Worldwide Interoperability for Microwave Access (WiMAX) that complies with IEEE802.16 [5] has received a lot of attention due to its potential in terms of wide communication area and high-speed data transmission. In particular, mobile WiMAX based on IEEE802.16e [6] is a very attractive system for mo-

\footnotetext{
Manuscript received December 9, 2009.

Manuscript revised March 17, 2010.

${ }^{\dagger}$ The authors are with NTT Access Service Systems Laboratories, NTT Corporation, Yokosuka-shi, 239-0847 Japan.

${ }^{\dagger}$ The author is with Faculty of Engineering, Nippon Institute of Technology, Saitama-ken, 345-8501 Japan.

${ }^{\dagger+\dagger}$ The author is with NTT East Corporation, Tokyo, 141-0022 Japan.

$+\dagger^{\dagger \dagger}$ The author is with Niigata University, Niigata-shi, 950-2181 Japan.

${ }^{+t+\dagger}$ The author is with Iwate University, Morioka-shi, 020-8551 Japan.
}

DOI: 10.1587/transcom.E93.B.2053 bile use, because mobile WiMAX provides the high speed transmission for mobile users.

The MWR has the functions of WLAN Access Point (AP) and of WiMAX Mobile Station (MS). The WLAN Stations (STAs) are connected to the AP in the MWR via an 802.11 link. Hence, the MWR converts media between IEEE802.11 and 802.16e. This fact enables the WLAN STAs to connect to the backbone IP network. However, the chassis of the MWR must be downsized because it is assumed to be carried in outdoor. Therefore WLAN and WiMAX devices are required to be installed next to each other in these MWRs. Moreover, the assigned frequency bands of WLAN and WiMAX are 2.4 and $2.5 \mathrm{GHz}$, respectively. This situation triggers mutual system interference between the two wireless devices and system throughput of the wireless relay is greatly degraded [7].

There are a lot of prior studies concerning MWRs that use WLANs and WiMAX [8]-[11], however, they concentrated on handover between WLAN and WiMAX. On the other hand, prior studies [12]-[14] proposed a resource management scheme for a hybrid network that bridged WLAN and WiMAX. However, the problem concerning mutual system interference was not considered. Although the studies [15], [16] analyzed the effect of mutual system interference in a heterogeneous network of WLAN, Bluetooth and/or WiMAX in detail, no interference avoidance technique was introduced. There are a lot of schemes that can suppress interference in the PHY layer [17]; however, hardware modification of existing WLAN terminals is required and signal processing is complex when using the interference suppression.

This paper proposes a novel interference avoidance technique that achieves a simple implementation using only MAC protocol. The proposed scheme synchronizes the transmission and the reception timing of WiMAX and WLAN and avoids the case where interference is generated in the MWR. Mutual system interference is generated when WiMAX device of WAN transmits signals while WLAN device of LAN receives signals. In the case when WLAN transmits signals while WiMAX receives signals, mutual system interference is generated in the same way. Ordinarily, it is difficult to synchronize transmission and reception timing of WLAN with WiMAX because Carrier-Sense Multiple Access (CSMA) adapted to IEEE802.11 WLAN is based on the random access procedure. On the other hand, Power Save Multi-Poll (PSMP) is regulated in order to save 
power consumption of WLAN in IEEE802.11n [18]. PSMP easily allows WLAN AP to control the transmission and reception timing of STAs. Hence, the proposed scheme enables interference avoidance between WLAN and WiMAX by applying PSMP.

The remainder of this paper is organized as follows. In Sect. 2, we describe the throughput degradation due to of mutual system interference using experimental equipment. The conventional schemes are introduced in Sect. 3. Sect. 4 proposes the interference avoidance technique using IEEE802.11n PSMP and we propose the channel allocation scheme. The effectiveness of the proposed scheme when considering actual WLAN and WiMAX scenario is verified in Sect. 5.

\section{Throughput Degradation due to Mutual System In- terference}

This section describes the condition that the mutual system interference is generated and the experiment conducted to verify the issue raised by the mutual system interference between WLAN and WiMAX.

Figures 1 and 2 illustrates the concept of WLANWiMAX relay via the MWR and simplified configuration of the MWR respectively. MWRs are expected to implement wireless devices into a size that is smaller than the business card. Therefore, the devices of WLAN and WiMAX are located at adjacent spaces, respectively. In addition to the condition, the emission of the spectrum mask leaks outside the band and caused mutual system interference when the frequency band of WLAN and WiMAX is adjacent. Figure 3 shows this problem conceptually. Here, we assume that the issue of mutual system interference generated in the

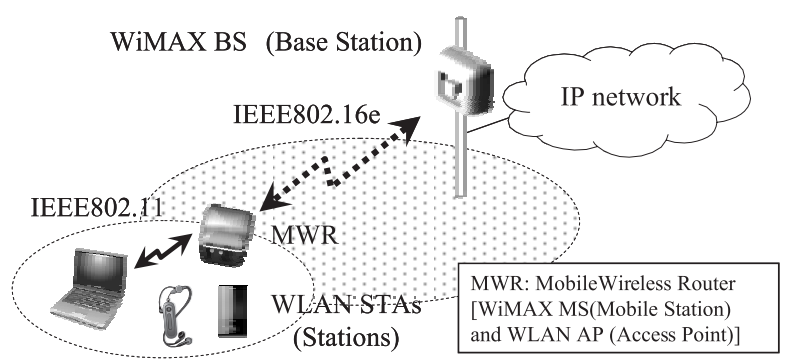

Fig. 1 Concept of WLAN-WiMAX relay via the MWR.

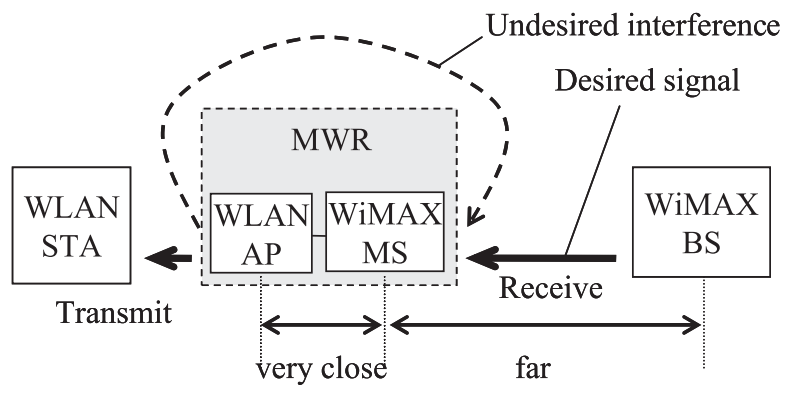

Fig. 2 Simplified MWR configuration. circuit in the MWR is another problem for the future work. In this paper, we concentrate on the issue of mutual system interference caused in the air.

To verify this problem practically, we conducted the experiment using WLAN AP, STA, WiMAX base station (BS) and MS. Figure 4 illustrates the block diagram of mutual system interference of the experiment. The experiment focuses on the throughputs of WLAN and WiMAX.

In order to precisely observe the power degradation caused by mutual system interference, we used wired links with the same attenuators as wireless links. That is, the attenuators degrade signal power by an amount equal to the free-space path loss yielded by the distance between wireless devices; fading is not considered in the experimental system. The parameters of the experiment are shown in Table 1. Besides, the standards of WLAN and WiMAX comply with IEEE802.11g and IEEE 802.16e respectively. The throughput characteristics of WiMAX and of WLAN are shown in Figs. 5 and 6, respectively. Figure 5 indicates that the downlink throughput of WiMAX began to degrade when the distance between WLAN AP and WiMAX MS were $10 \mathrm{~cm}$ apart, the downlink throughput halved at $5 \mathrm{~cm}$, and connection lost at approximately $1 \mathrm{~cm}$. Figure 7 illus-

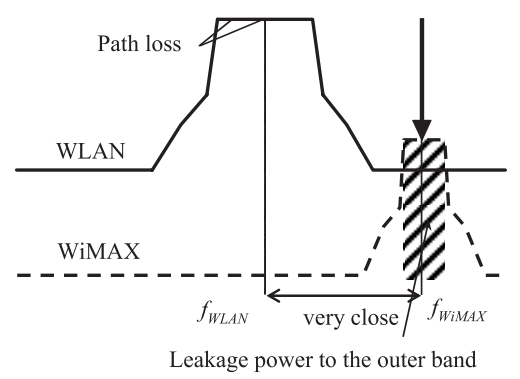

Fig. 3 The concept of mutual system interference.

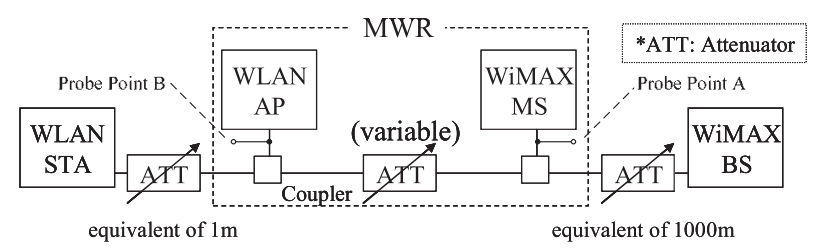

Fig. 4 Block diagram of mutual interference.

Table 1 Parameters of the experiment.

\begin{tabular}{|c|c|}
\hline Transmission Power of the WLAN AP / the STA & $20[\mathrm{dBm}]$ \\
\hline Transmission Power of the WiMAX MS & $27[\mathrm{dBm}]$ \\
\hline Transmission Power of the WiMAX BS & $45[\mathrm{dBm}]$ \\
\hline Distance between WLAN STA and WLAN AP & $1[\mathrm{~m}]$ \\
\hline Distance between WiMAX MS and WiMAX BS & $1000[\mathrm{~m}]$ \\
\hline Distance between WLAN AP and WiMAX MS & $0.01-0.6[\mathrm{~m}]$ \\
\hline WiMAX Scheduling Type & UGS \\
\hline WiMAX DL/UL Ratio & $32: 15$ \\
\hline WiMAX Maximum Received Traffic Rate & $\begin{array}{c}\text { DL: } 2[\mathrm{Mbit} / \mathrm{s}] \\
\text { UL:1 }[\mathrm{Mbit} / \mathrm{s}]\end{array}$ \\
\hline
\end{tabular}




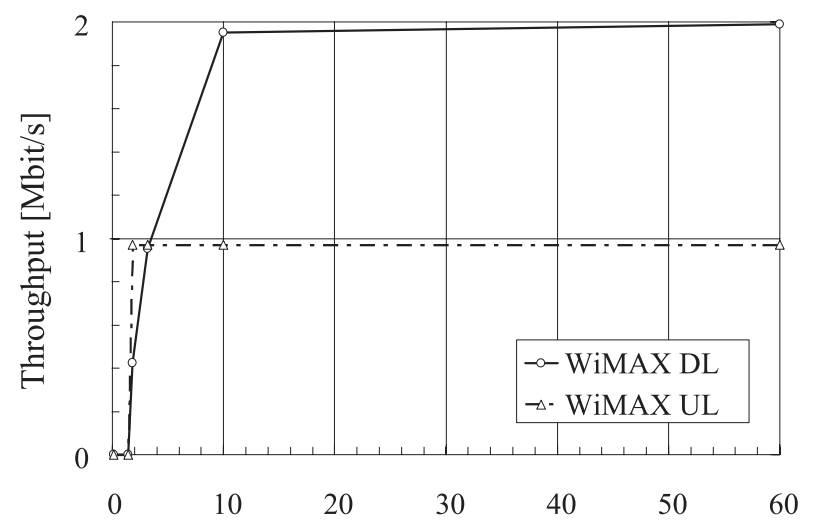

Fig. 5 Uplink/Downlink throughput of WiMAX.

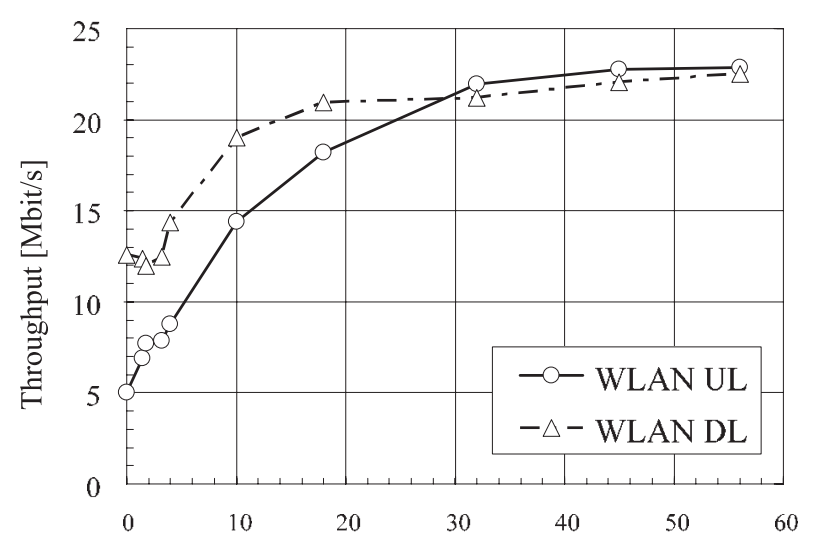

Fig. 6 Uplink/Downlink throughput of WLAN.

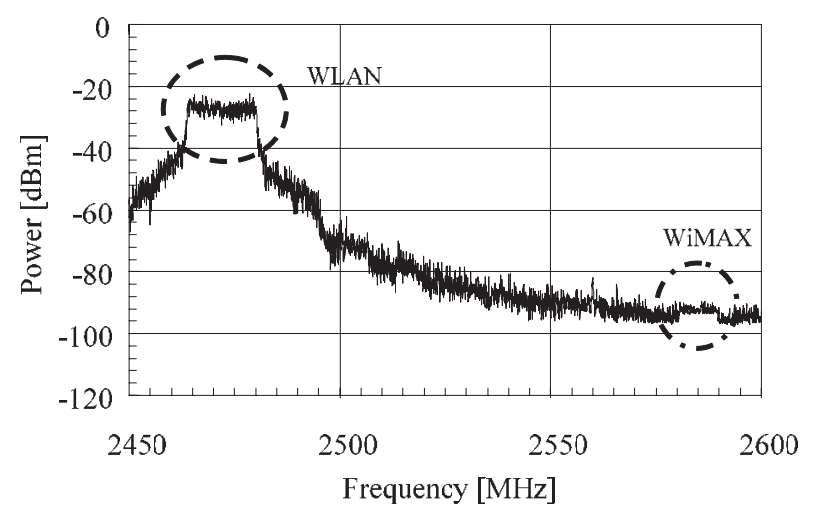

Fig. 7 Waveform of WLAN and WiMAX signals in downlink.

trates the waveform of interference WLAN signals and desired WiMAX signals that observed at $1 \mathrm{~cm}$ distance. In the observation point, WiMAX MS is receiving DL Burst while WLAN AP is transmitting signals for WLAN STA. Point "A" in Fig. 4 is the probe point of this waveform. From Fig. 7, desired WiMAX signal is almost buried in the emission of undesired WLAN signal.

Therefore, it is clear that WLAN interference degrades WiMAX throughput. Figure 6 also indicates that WLAN uplink throughput began to degrade at the separation distance of $32 \mathrm{~cm}$; a $70 \%$ throughput reduction occurred at

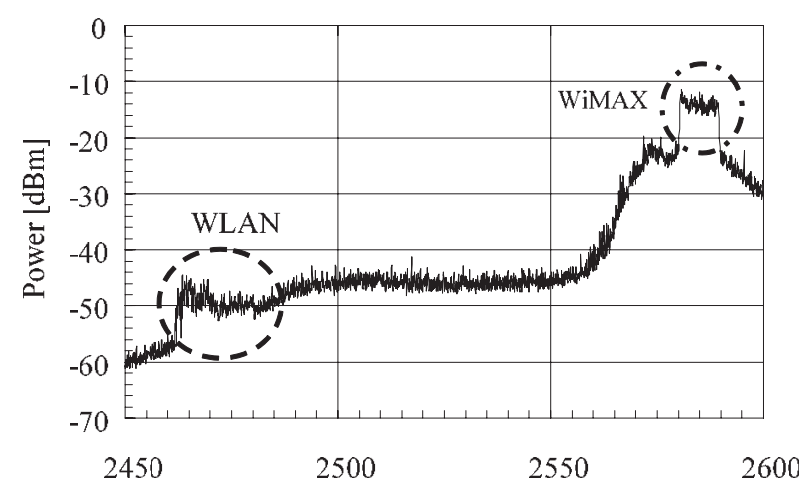

Fig. 8 Waveform of WLAN and WiMAX signals in uplink.

$5 \mathrm{~cm}$. Figure 8 illustrates the waveform of desired WLAN signals and interference WiMAX signals that observed at $1 \mathrm{~cm}$ distance. In the observation point, WLAN AP is receiving signals from WLAN STA while WiMAX MS is transmitting UL Burst. Point " $\mathrm{B}$ " in Fig. 4 is the probe point of this waveform. As well as Fig. 7, Fig. 8 indicates that desired WLAN signal severely disturbed from the emission of undesired WiMAX signal. Therefore, WiMAX interference degrades WLAN throughput in both the uplink and downlink. The reason why not only WLAN UL throughput but also WLAN DL throughput decreases is as follows;

When WLAN STA receives the signal correctly from WLAN AP, WLAN STA casts an ACK (Acknowledgement) frame to WLAN AP. This ACK frame is carried in a signal sent in the WLAN UL direction. Therefore, this ACK frame is affected by the mutual system interference from WiMAX UL signals. The throughput of WLAN DL decreases as a result so that WLAN AP, which was not able to receive ACK frames properly resends, DL signals to WLAN STA. Moreover, WLAN AP transmission was halted due to the carrier sense function which uses the CSMA algorithm.

\section{Conventional Operation for MWR and Interference Avoidance Scheme and Issues}

This section introduces conventional operation and interference avoidance scheme for MWRs and their issues. MAC protocol procedures are detailed below.

\subsection{Conventional Operation for MWRs}

The conventional operation is shown hereafter. WLAN and WiMAX perform their normal transmission sequences independently. We define this scheme as Autonomous Distributed Control (ADC) as shown in Fig. 9.

In ADC, no synchronization between WLAN and WiMAX is performed. Although the conventional scheme using the ADC is simple, mutual system interference occurs if one of the wireless devices in the MWR is receiving signals while the other is transmitting signals. Particularly if mutual system interference occurs while receiving WiMAX control signals such as preamble, FCH (Frame 


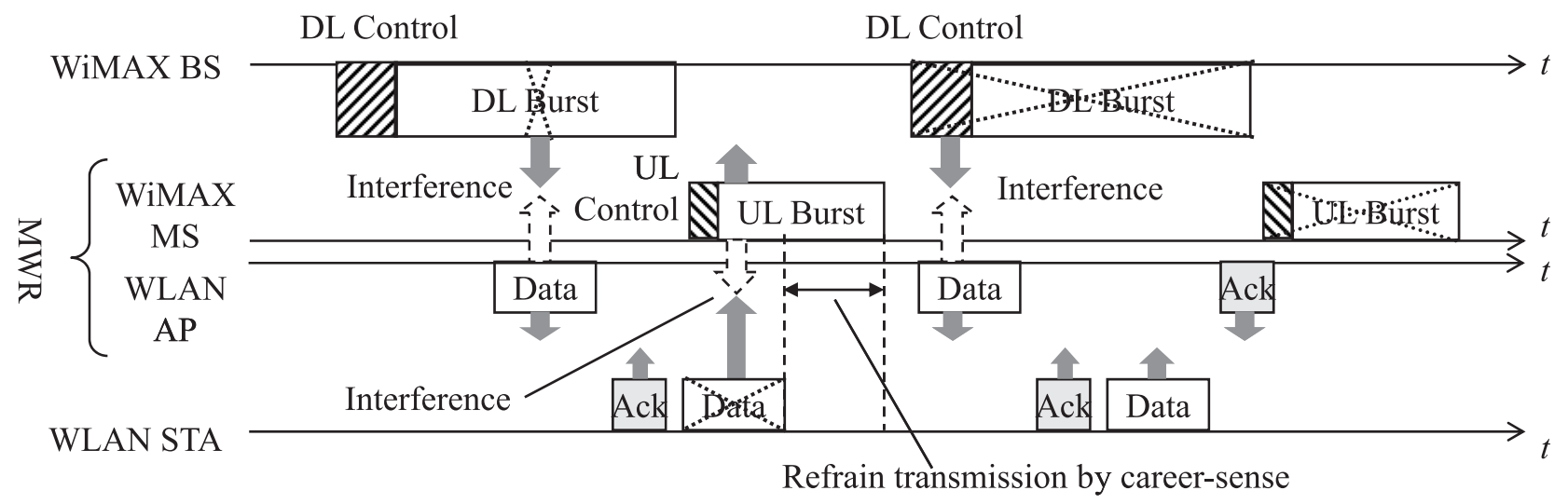

Fig. 9 Conventional ADC operation.

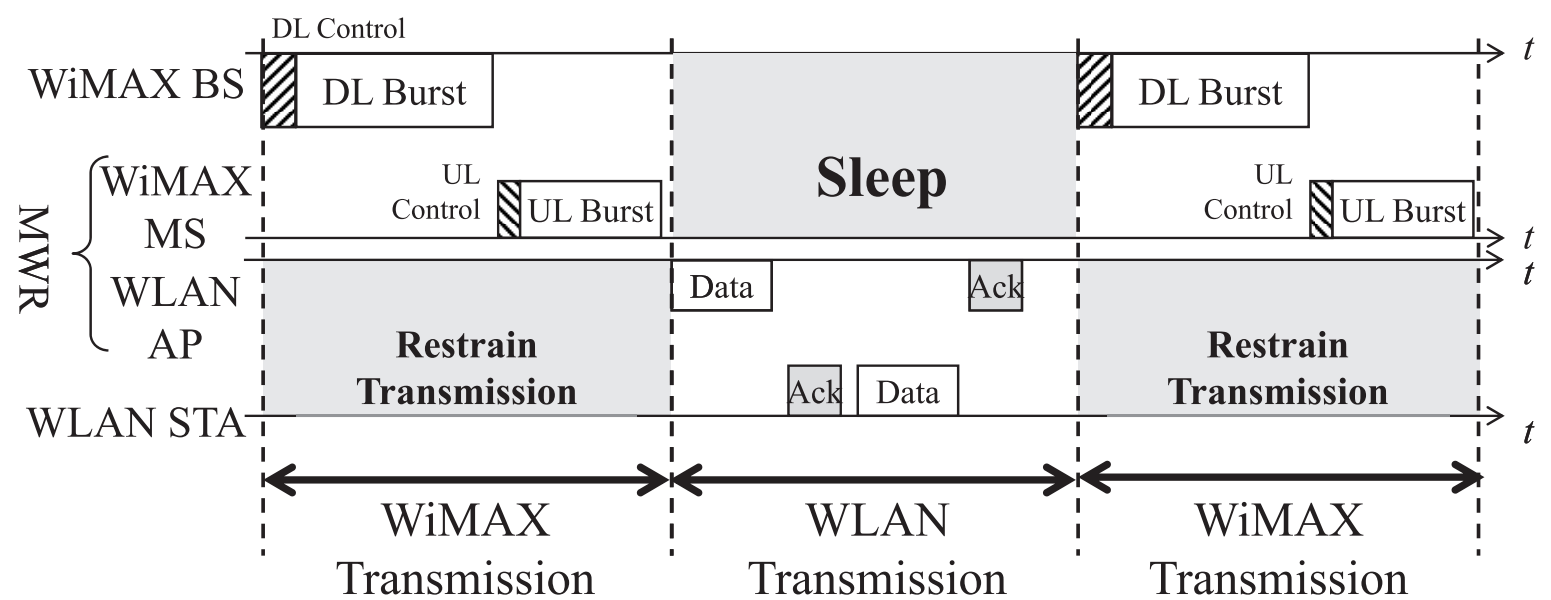

Fig. 10 Conventional TDD operation.

Control Header), Downlink (DL)-MAP and Uplink (UL)MAP, throughput is severely degraded by the corruption of entire WiMAX frames.

\subsection{Conventional Interference Avoidance Scheme: TDD}

To avoid mutual system interference, a scheme by which WLAN and WiMAX can coexist, is being discussed in WiMAX Forum [19]. That scheme leverages the sleep mode function specified in IEEE802.16e. In the sleep mode, WiMAX BS divides the transmission period into sleeping period and listening period. Listening period is the time for normal transmission, and the sleeping period halts all transmission to cut WiMAX MS power consumption. In this scheme, WLAN AP executes its normal transmission during WiMAX sleeping period, on the other hand, all WLAN devices refrain from transmission during the WiMAX listening period as shown in Fig. 10. Time Division Duplex (TDD) scheme prevents mutual system interference. However, it halves the throughput of each wireless system.

\section{Proposed Mutual System Interference Avoidance Scheme}

The proposed scheme avoids mutual system interference without great reducing throughput. The proposed scheme leverages PSMP function specified in IEEE 802.11n. PSMP was designed for WLAN STA power saving; however we utilize PSMP for interference avoidance. First, the operation of PSMP is explained, and then the concept of the proposed scheme is introduced. Finally, the whole proposal is described in detail.

Figure 11 depicts PSMP procedure realized by IEEE802.11n. In PSMP, WLAN AP broadcasts a PSMP frame to all WLAN STAs. This PSMP frame contains information about the timing of transmission and of reception for STAs. Besides, the PSMP frame indicates the length of Downlink Phase and Uplink Phase. PSMP procedure enables AP to divide transmission period into Downlink Phase and Uplink Phase.

In Downlink Phase, only WLAN AP can transmit signals to WLAN STAs and these STAs are prohibited from sending any signals including ACK frames. On the other hand, WLAN STAs can transmit during Uplink Phase. In PSMP sequence, MAC Protocol Data Units (MPDUs) or Aggregate MAC Protocol Data Units (A-MPDUs) are allowed to use. Moreover, they are delivered in the interval of Short Interframe Space (SIFS) or Reduced Interframe Space (RIFS). On the other hand, WLAN STAs and AP send an ACK, Block ACK or Multiple TID Block Ack (MTBA) in 


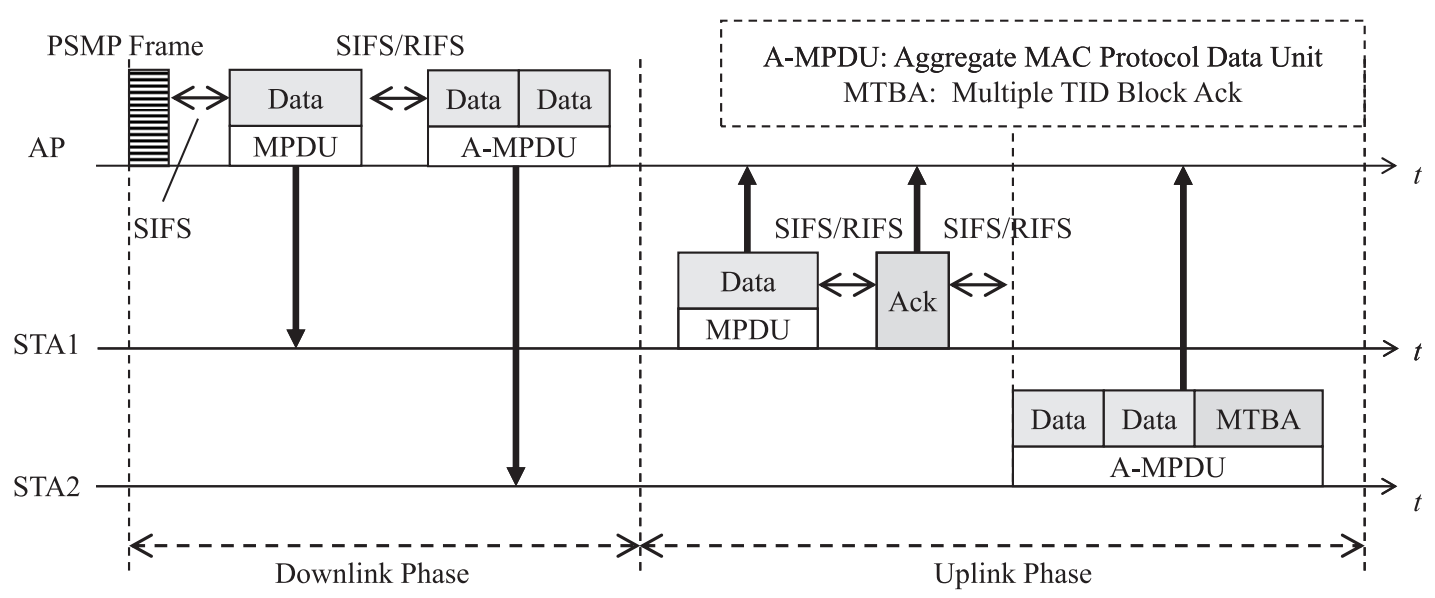

Fig. 11 PSMP Sequence.

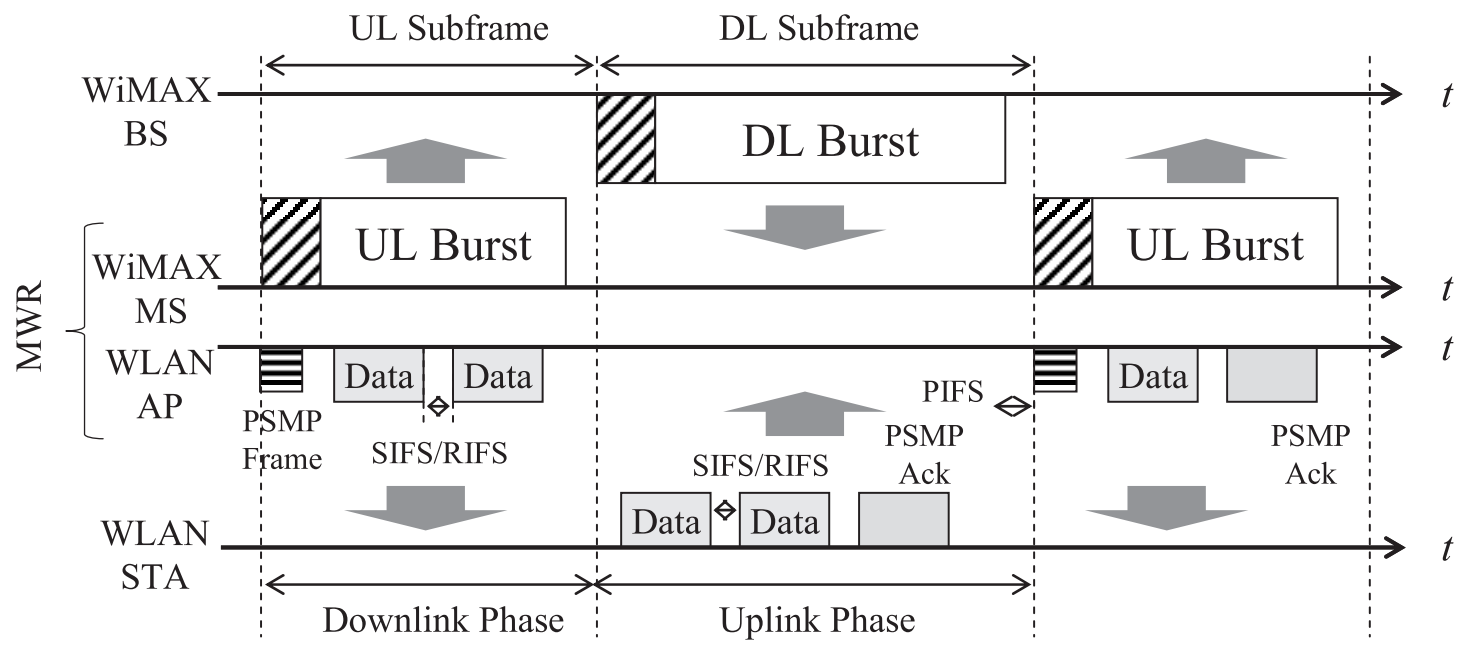

Fig. 12 Basic concept of the proposed method.

their transmission period (Downlink Phase for AP, Uplink Phase for STAs) for data received during prior Downlink Phase or Uplink Phase. A period that consists of Downlink Phase and Uplink Phase is called a PSMP sequence. As a result, WLAN STAs only receive or transmit signals during assigned period which is indicated by PSMP frame. The concept of the proposed scheme is to make the PSMP sequence as long as a WiMAX frame. That is, the proposed scheme makes the length of WLAN Uplink Phase equal to the length of WiMAX DL Subframe. The length of WLAN Downlink Phase is made equal to the length of WiMAX UL Subframe as well. The basic operation of the proposed scheme is shown in Fig. 12. Accordingly, the basic operation enables the MWR to transmit signals only in WLAN Downlink Phase, and to receive signals only in WLAN Uplink Phase. That is, the MWR eliminates mutual system interference which realizes highly effective relay transmission.

On the other hand, in the Mobile WiMAX profile [20], the ratio of the number of OFDM symbols of DL to that of UL has been decided. According to the profile, the length of DL Subframe is longer than UL Subframe. In Japan and many other countries, the dominant numbers of OFDM Symbols in DL and in UL are set at 32 and 15, respectively. In this paper, the length of DL Subframe and UL Subframe complies with above system profile. Therefore, the proposed scheme is more effective than conventional ADC or TDD; however, system throughput might be degraded by the difference in traffic between WiMAX and WLAN. In other words, the transmitted WLAN traffic might be less than WiMAX traffic because the transmission period of WLAN AP (WLAN Downlink Phase) is limited by the WiMAX UL Subframe period as shown in Fig. 13. In Fig. 13, the horizontal axis represents time, and the vertical axis means actual transmission rate of each wireless system including loss of overhead (for example, Inter Frame Space (IFS) for WLAN, Frame Control Header (FCH), DL-MAP and UL-MAP for WiMAX).

Our solution is to add a scheduling scheme to the basic proposed scheme. The scheduling scheme modifies the length of WLAN Downlink Phase in order to make the gap of the amount of traffic between WiMAX downlink and 


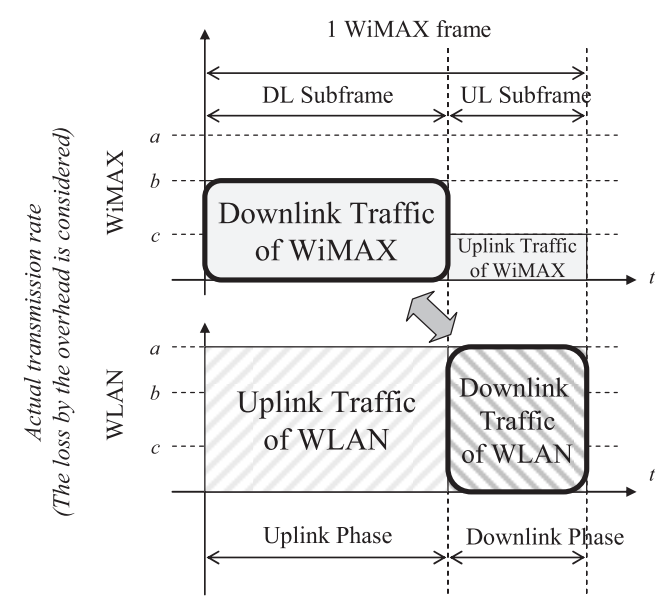

Downlink Traffic of WiMAX > Downlink Traffic of WLAN

Fig. 13 Key issue with the basic concept.

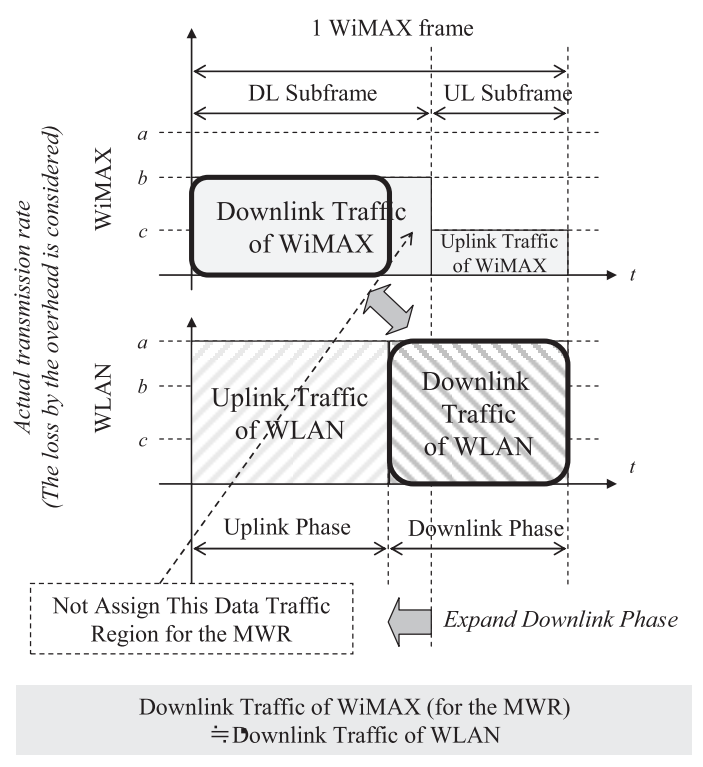

Fig. 14 The scheduling method for downlink.

WLAN downlink minimum. First, the scheduler expands the WLAN Downlink Phase to match the amount of WLAN downlink traffic and WiMAX downlink traffic as shown in Fig. 14. At this time, the MWR requests WiMAX BS not to allocate WiMAX DL Burst for the MWR after the beginning of WLAN Downlink Phase using the message of Bandwidth Request Header. The reasons for this are that the WiMAX DL Burst and the transmission in WLAN Downlink Phase after the period might trigger mutual system interference. Second, as well as the downlink scheduling, the optimal length for WLAN Uplink Phase is decided. The expansion of the WLAN Downlink Phase equals the reduction of the WLAN Uplink Phase because the total length of WiMAX frame is constant. Therefore, excessive expansion of the WLAN Downlink Phase reduces uplink traffic that needs for WiMAX UL Burst. This causes the degradation of uplink throughput. To prevent this throughput

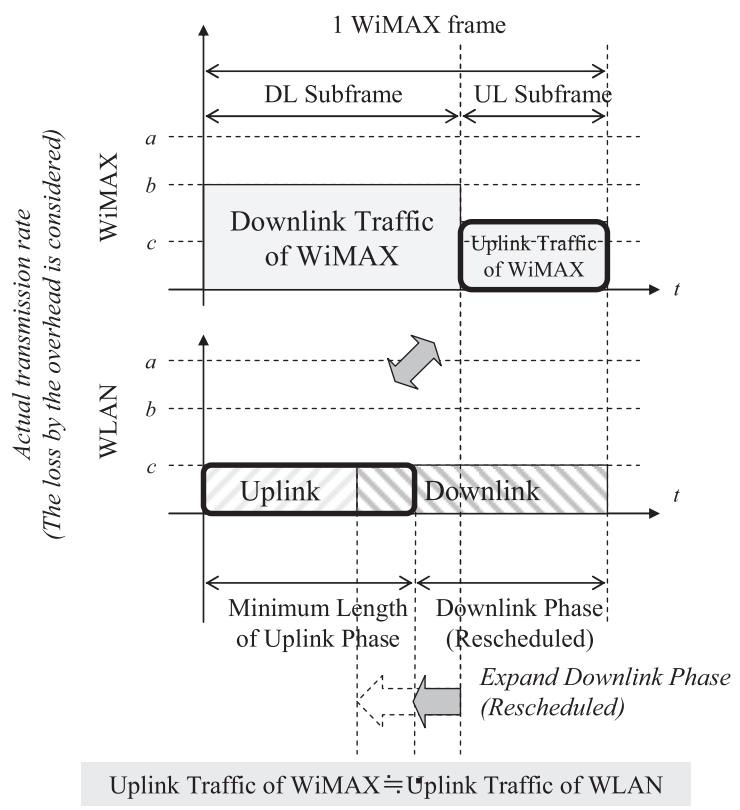

Fig. 15 The scheduling method for uplink.

degradation, the minimum length of WLAN Uplink Phase is introduced. Accordingly, the beginning of WLAN Downlink Phase is rescheduled to match the end of the minimum length of WLAN Uplink Phase. Following this reasoning, the minimum length of WLAN Uplink Phase is decided so as to match the amount of WLAN uplink traffic andWiMAX uplink traffic as shown in Fig. 15. Hence, system throughput is improved by this scheduling scheme. Analysis details are presented in the Appendix.

As a consequence, this scheduling change improves the throughput via the WLAN-WiMAX relay system.

\section{Performance of Proposed and Conventional Sche- mes}

To clarify the performance of the proposed scheme, system throughputs of WiMAX BS and WLAN STA with the conventional and proposed schemes are evaluated by computer simulations. As the simulation conditions, we assume that if any packets (in the case of WLAN) or any OFDM symbols (in the case of WiMAX) suffer mutual system interference, those packets or symbols should be discarded. Moreover, we assume that there is no interference between the MWR and WiMAX BS or WLAN STA. These assumptions are reasonable given that the MWRs and WiMAX BS or WLAN STA will be very far apart compared to the distance between WLAN AP and WiMAX MS in the MWR. Moreover, downlink and uplink traffic flows occur simultaneously.

WLAN parameters of conventional schemes and of the proposed scheme comply with IEEE802.11g and IEEE802.11n legacy mode respectively. As well, WiMAX parameters comply with IEEE802.16e-2005 standard. Under this arrangement, the offered traffic (uplink and downlink) represents the traffic generated from the transmission 


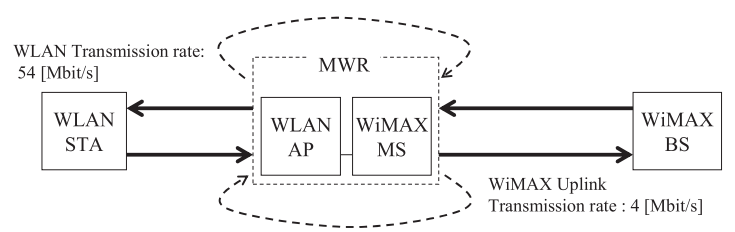

Fig. 16 The simulation model.

Table 2 The simulation parameters.

\begin{tabular}{|c|c|c|c|c|c|}
\hline & \multicolumn{5}{|c|}{ Parameters } \\
\hline & $\begin{array}{r}\text { WLAN } \\
T R \\
{[\mathrm{Mbit} / \mathrm{s}]}\end{array}$ & $\begin{array}{c}\text { WLAN Uplink } \\
\text { OT } \\
\text { [Mbit/s] }\end{array}$ & $\begin{array}{c}\text { WiMAX } \\
\text { Uplink TR } \\
\text { [Mbit/s] }\end{array}$ & $\begin{array}{c}\text { WiMAX } \\
\text { Downlink OT } \\
\text { [Mbits] }\end{array}$ & $\begin{array}{c}\text { WiMAX } \\
\text { Downlink TR } \\
\text { [Mbit/s] }\end{array}$ \\
\hline $\begin{array}{l}\text { Downlink } \\
\text { Throughput }\end{array}$ & 54 & 30 & 4 & $3.7-18.7$ & $3.7-18.7$ \\
\hline $\begin{array}{l}\text { Uplink } \\
\text { Throughput }\end{array}$ & 54 & $1-10$ & 4 & 18.7 & 18.7 \\
\hline & & Transmis & Rate & OT:Offere & raffic \\
\hline
\end{tabular}

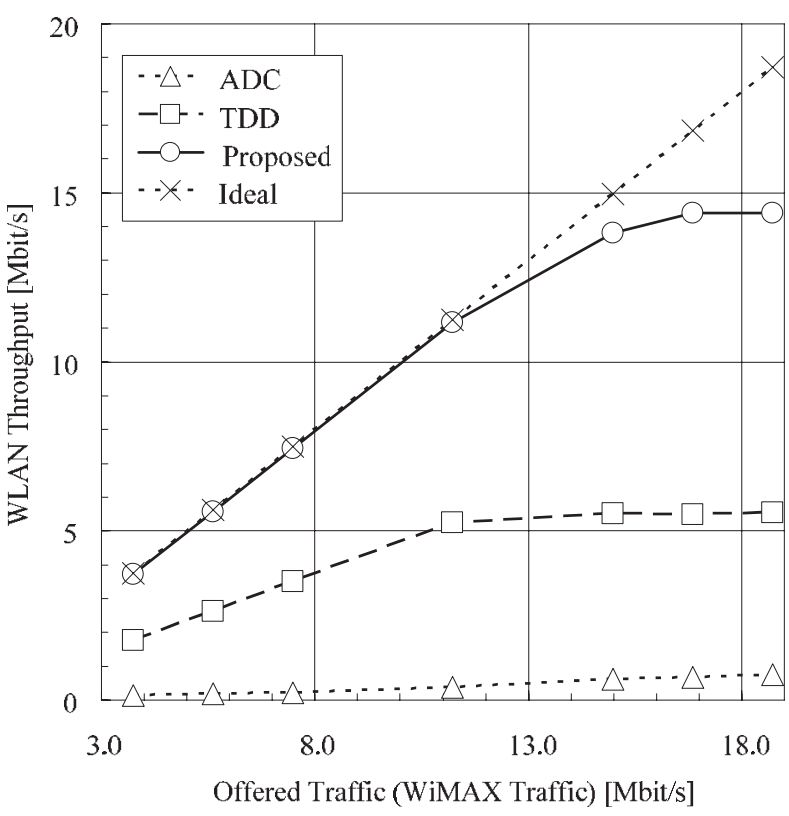

Fig. 17 Downlink throughput characteristics in the proposed and conventional methods.

edge. Figure 16 shows the simulation model; the simulation parameters are given in Table 2.

The comparison of proposed and conventional schemes is performedunder the above-mentioned conditions.

Figures 17 and 18 shows the received downlink throughput characteristics of WLAN STA and the received uplink throughput characteristics of WiMAX. In Fig. 17, ADC yields little throughput because most of data traffic are lost by the intermittent mutual system interference. TDD limits the throughput to less than half the ideal value even though it prevents mutual system interference. In particular, uplink and downlink share the communication period already halved by time division done with WiMAX. Therefore, TDD is able to obtain little throughput after the point of $11 \mathrm{Mbit} / \mathrm{s}$ in the offered traffic. On the other hand, the proposed scheme attained 19.2 times throughput from ADC and

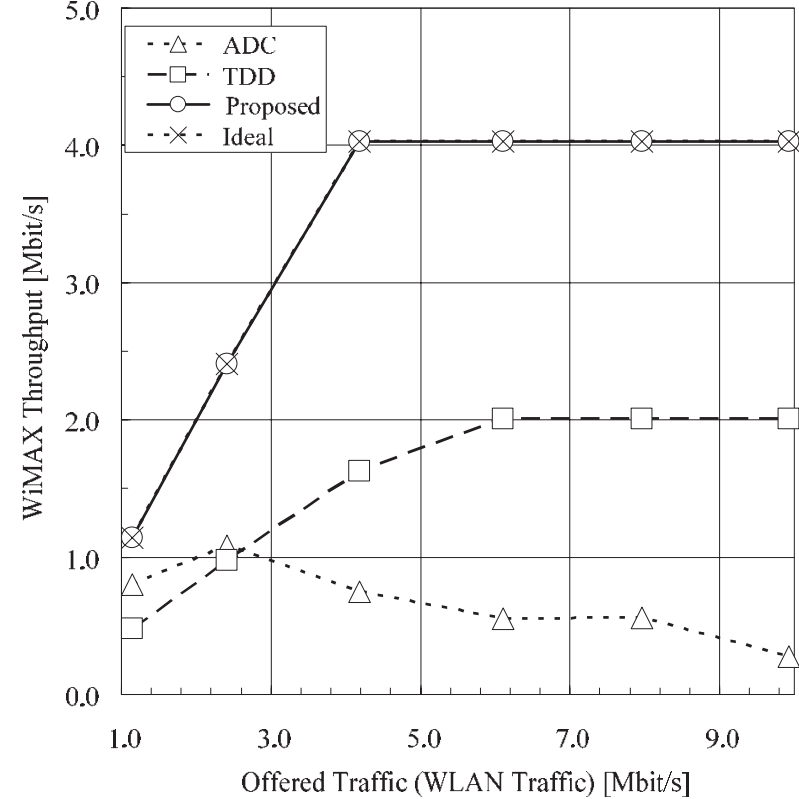

Fig. 18 Uplink throughput characteristics in the proposed and conventional methods.

2.6 times throughput from TDD at the point of $17 \mathrm{Mbit} / \mathrm{s}$ in the offered traffic. Though the throughput characteristic of the proposed scheme is saturated after the point of $11 \mathrm{Mbit} / \mathrm{s}$ in the offered traffic, the reason is there is no room to expand WLAN Downlink Phase. In other words, optimal scheduling that WiMAX downlink traffic and WLAN downlink traffic is almost same has been achieved after that point.

In Fig. 18, the reason why little throughput has yielded in ADC and TDD is the same as the case of downlink. On the contrary, the proposed scheme obtained nearly ideal throughput. The proposed scheme obtains 14.4 times throughput from ADC and twice from TDD at the point of $10 \mathrm{Mbit} / \mathrm{s}$ in the offered traffic. Though the throughput characteristic of the proposed scheme and ideal is saturated after the point of $4 \mathrm{Mbit} / \mathrm{s}$ in the offered traffic, the reason is quite different from the uplink case. That is, because the boundary is up to $4 \mathrm{Mbit} / \mathrm{s}$ of WiMAX uplink throughput, it becomes impossible to exceed this value.

\section{Conclusion}

This paper proposed a novel interference avoidance technique on MAC protocol. The proposed scheme synchronizes the transmission and the reception timing of WiMAX and WLAN using IEEE802.11n PSMP and avoids mutual system interference. In addition to this proposition, we proposed the scheduling scheme to eliminate the gap of traffic that improves throughput characteristic via the WiMAXWLAN relay system. F irst, we described that the impact of mutual system interference between WLAN and WiMAX units was clarified by the experiment. We showed that the conventional scheme of ADC allows mutual system interference to significantly degrade throughput, and the addi- 
tion of TDD, while preventing mutual system interference, halves the throughput since it shares the communication period. On the other hand, the proposed scheme achieves good throughput; it eliminates mutual system interference by careful scheduling without wasting the communication period. The computer simulation confirms its good performance. The proposed scheme succeeded in obtaining about 20 times throughput compared with ADC and about 3 times throughput compared with TDD.

This paper discussed the scenario of one MWR connected to WiMAX BS as an initial investigation. As future work, we must extend the scheduling algorithm to improve throughput performance when multiple MWRs are connected to WiMAX BS; of the goal is to ensure that the optimal WiMAX DL Burst allocation is achieved. We note that the time position of DL Burst for each MWR in WiMAX frame may limit the length of WLAN Downlink phase, and this limitation might degrade system performance. Even without this extended algorithm, the proposed scheme ensures that mutual interference problem is suppressed. That is, the basic concept of the proposed scheme, which is to make the PSMP sequence as long as the WiMAX frame, is effective.

\section{Acknowledgement}

We are deeply grateful to Mr. Kengo Nagata and Mr. Makoto Umeuchi whose enormous support and insightful comments were invaluable during the course of our study.

\section{References}

[1] IEEE Standard for Local and Metropolitan Area Networks Part 11: Wireless LAN Medium Access Control (MAC) and Physical Layer (PHY) Specifications, 2008.

[2] V.P. Kafle, E. Kamioka, and S. Yamada, "MoRaRo: Mobile routerassisted route optimization for network mobility (NEMO) support," IEICE Trans. Inf. \& Syst., vol.E89-D, no.1, pp.158-170, Jan. 2006.

[3] E. Wallenius, T. Hamalainen, T. Nihtila, J. Puttonen, and J. Joutsensalo, "Simulation study on 3G and WLAN inter-working," IEICE Trans. Commun., vol.E89-B, no.2, pp.446-459, Feb. 2006.

[4] S.K. Sadhukhan, S. Mandal, and D. Saha, "A methodology for WLAN planning under 3G network," Global Mobile Congress 2009, pp.1-6, Oct. 2009.

[5] IEEE Standard for Local and Metropolitan Area Networks Part 16: Air Interface for Fixed Broadband Wireless Access Systems, 2005.

[6] 802.16TGe-2005 Standard: Physical and Medium Access Control Layers for Combined Fixed and Mobile Operation in Licensed Bands, Feb. 2006.

[7] N.J. Thomas, M.J. Willis, and K.H. Craig, "Analysis of co-existence between IEEE 802.11 and IEEE 802.16 systems," 2006 3rd Annual IEEE Communications Society on Sensor and Ad Hoc Communications and Networks, SECON'06, vol.2, pp.615-620, Sept. 2006.

[8] X. Chen, H. Zhou, Y. Qin, and H. Zhang, "Multi-interfaced mobile router scheme and enhanced path selection algorithm," International Conference on Telecommunications, ICT 2008, pp.16-19, June 2008.

[9] W. Liao and K. Chen, "Supporting vertical handover between universal mobile telecommunications system and wireless LAN for real-time services,” IEEE Trans. Commun., vol.2, no.1, pp.75-81, Jan. 2008.
[10] W. Choi, T.S. Shon, H.H. Choi, and Y. Lee, "Designing a novel unlicensed nomadic access relay station in IEEE 802.16-based wireless access networks," Vehicular Technology Conference 2007, VTC2007-Spring, pp.2961-2965, April 2007.

[11] J.C. Lee and H.J. Kim, "The design of mobile router supporting heterogeneous access networks and IPv4 traversal," ICACT2007, Feb. 2007.

[12] D. Niyato and E. Hossain, "Integration of IEEE 802.11 WLANs with IEEE 802.16-based multihop infrastructure mesh/relay networks: A game-theoretic approach to radio resource management," IEEE Netw., vol.21, no.3, May-June 2007.

[13] J. Nie, X. He, Z. Zhou, and C. Zhao, "Communication with bandwidth optimization in IEEE 802.16 and IEEE 802.11 hybrid networks," Proc. ISCIT2005, Oct. 2005.

[14] G.M. Lee, B.C. Jeon, S. Heo, H. Kim, J. Jin, and S. Lee, "Load balancing scheme and implementation in multihoming mobile router," NOMS2008, April 2008.

[15] A. Stranne, O. Edfors, and B.A. Molin, "Energy-based interference analysis of heterogeneous packet radio networks," IEEE Trans. Commun., vol.54, no.7, July 2006.

[16] S. Suansook, S. Aramvith, and P. Prapinmongkolkarn, "Comparative performance analysis of WiMAX and WLAN with WPAN coexistence in UL band," Proc. ISPACS2007, Nov.-Dec. 2007

[17] W.S. Chen, Y.C. Chang, H.T. Chen, F.S. Chang, and H.C. Su, "Novel design of printed monopole antenna for WLAN-WiMAX applications," APS2007 IEEE, June 2007.

[18] IEEE Standard for local and Metropolitan Area Networks Part 11: Wireless LAN Medium Access Control (MAC) and Physical Layer (PHY) Specifications, Amendment 5 Enhancements for Higher Throughput, Oct. 2009.

[19] J. Lei, Y. Yunsong, W. Xuyong, and J. Segev, "Sleep mode timing amendment proposal," IEEE 802.16 Working Group Letter Ballot Recirc \#26a, Jan. 2008., http://www.ieee802.org/16/maint/contrib/ C80216maint-08_035r5.doc

[20] WiMAX Forum Mobile System Profile Release 1.0 Approved Specification (Revision 1.2.2), Nov. 2006.

\section{Appendix: Calculation for the Optimal WLAN Down- link Phase}

Details of the theoretical calculation used in Sect. 4 of the proposed scheme are explained here. Figure A. 1 illustrates how to calculate for the optimal WLAN Downlink Phase.

First, on the basic proposed scheme, we define the amount of traffic that received at WiMAX MS as $C_{x}$ in a

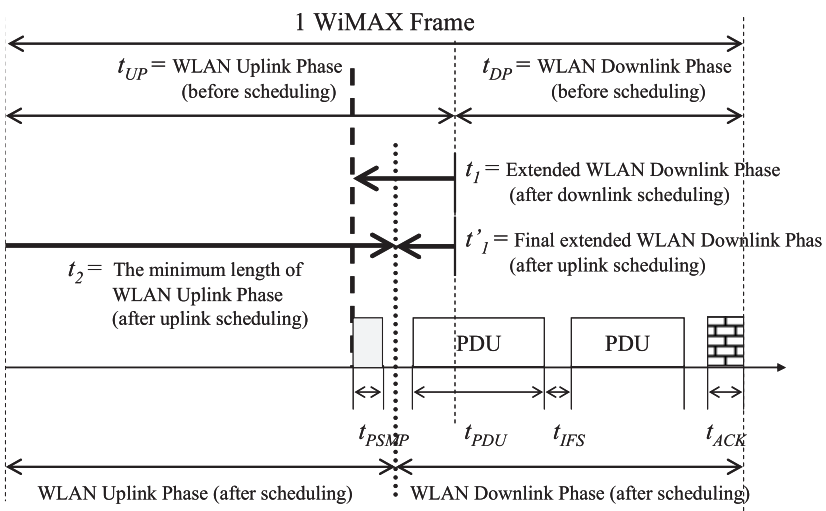

Fig. A. 1 Calculation for the optimal WLAN Downlink Phase. 
WiMAX frame. As well, we also define the amount of traffic that transmitted at WLAN AP as $C_{w}$ in a WiMAX frame. Then traffic difference $C_{e x}$ shown as (A-1) will not be transferred to WLAN STAs.

$$
C_{e x}=C_{x}-C_{w}
$$

In addition, the number of all subcarriers that WiMAX DL Burst uses is $s$, the number of OFDM symbols that DL Burst allocated to the MWR is $L$, and modulation bit number is $m . C_{x}$ is represented as (A.2).

$$
C_{x}=s \times L \times m \times e
$$

Variable $e$ is a coefficient of proportion of data payload in a DL Burst. We assume that the allocation of DL Burst is to use all subchannels, and to arrange in the time direction for simplification. We define that the number of WLAN packets that can be transmitted in WLAN Downlink Phase is $n_{w}$. We assume that the Ethernet frame in 1500 bytes is payload of WLAN packet hereafter. Payload of the Ethernet frame that doesn't come up to 1500 bytes is assumed to be $a$ as well. Therefore, $C_{w}$ is represented as (A.3)

$$
C_{w}=1500 \times 8 \times n_{w}+a \times 8
$$

Therefore, $n_{w}$ is given as follows.

$$
\begin{aligned}
& n_{w}=\text { floor }\left[t_{D P}-t_{O}\right] / t_{P} \\
& t_{O(a)}=t_{P S M P}+t_{A C K}+t_{P D U(a)}+t_{I F S} \\
& t_{P}=t_{P D U(1500)}+t_{I F S}
\end{aligned}
$$

The variables $t_{D P}$ and $t_{P D U}$ represent the length of the WLAN Downlink Phase before the scheduling and PDU length, respectively. $t_{P S M P}, t_{A C K}$, and $t_{I F S}$ represents each the length of PSMP frame, ACK, and IFS length.

Second, for our expanded scheduling scheme, we define the expanded length of WLAN Downlink Phase as $t_{1}$. The period $t_{1}$ is led from the following calculations. We assume that the number of all OFDM symbols in DL Burst is $L_{\max }$, and the length of an OFDM symbol is $l$. Therefore, the amount of traffic that WiMAX MS receives after the scheduling in a WiMAX DL Subframe is given by as $C_{x}^{\prime}$ shown below.

$$
C_{x}^{\prime}=C_{x}-\left\{s \times\left[\left(L_{\max }-L\right)+\operatorname{ceil}\left(\frac{t_{1}}{l}\right)\right] \times m \times e\right\}
$$

The amount of traffic that WLAN AP transmits after the scheduling is given by $C_{w}^{\prime}$.

$$
C_{w}^{\prime}=\left(1500 \times n_{w}^{\prime}+a^{\prime}\right) \times 8
$$

As well as before the scheduling, $n_{w}^{\prime}$ is the number of packets that have 1500 byte payload of Ethernet frames in WLAN Downlink Phase after the scheduling, and $a^{\prime}$ represents the payload of Ethernet frame whose size is less than 1500 bytes. Therefore, $n_{w}^{\prime}$ is given as follows.

$$
\begin{aligned}
& n_{w}=\text { floor }\left[\left(t_{1}+t_{D P}\right)-t_{O}\right] / t_{P} \\
& t_{O\left(a^{\prime}\right)}=t_{P S M P}+t_{A C K}+t_{P D U\left(a^{\prime}\right)}+t_{I F S}
\end{aligned}
$$

$$
t_{P}=t_{P D U(1500)}+t_{I F S}
$$

The scheduling scheme determines $t_{1}$ for which $C_{x}^{\prime}=C_{w}^{\prime}$. Therefore, $t_{1}$ is given by $(\mathrm{A} \cdot 12)$.

$$
t_{1}=\frac{s \cdot m \cdot e \cdot\left(2 L-L_{\max }\right)-1500 \cdot 8 \cdot\left(t_{D P}-t_{O\left(a^{\prime}\right)}\right) / t_{p}}{s \cdot m \cdot e / l+1500 \cdot 8 / t_{p}}
$$

Finally, the final expanded length of WLAN Downlink Phase $t_{1}^{\prime}$ is decided. To calculate $t_{1}^{\prime}$, the minimum length of WLAN Uplink Phase $t_{2}$ is decided as follows. We define the amount of traffic that transmitted from WiMAX MS as $G_{x}$ in a WiMAX frame. The number of all subcarriers that WiMAX UL Burst uses is $u$, the number of OFDM symbols that UL Burst allocated to the MWR is $J$, and modulation bit number is $i . G_{x}$ is represented as (A-13).

$$
G_{x}=u \times J \times i \times e
$$

Here, the quotient to divide $G_{x}$ by 1500 bytes is assumed to $n_{u}$ and the surplus is assumed to be $c$. Therefore, $t_{2}$ is represented as follows.

$$
\begin{aligned}
& t_{2}=t_{P} \times n_{u}+t_{R(c)} \\
& t_{R(c)}=t_{A C K}+t_{P D U(C)}+t_{I F S}
\end{aligned}
$$

We assume that $t_{U D}$ represent the length of the WLAN Uplink Phase before the scheduling. Accordingly, the final expanded length of WLAN Downlink Phase $t_{1}^{\prime}$ is decided as (A.16).

$$
t_{1}^{\prime}= \begin{cases}t_{U D}-t_{2} & \left(t_{2}<t_{U D}-t_{1}\right) \\ t_{1} & \left(t_{2} \geq t_{U D}-t_{1}\right)\end{cases}
$$

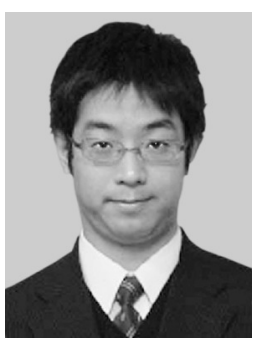

Akira Kishida received his B.S. and M.S. degrees from Tokyo University of Agriculture and Technology, Tokyo, Japan, in 2005 and 2007, respectively. He joined NTT Access Service Systems Laboratories in 2007. He is engaged in the research of interference avoidance technique on MAC protocol for multi-wireless systems such as between Wireless LAN and WiMAX. His current interests are high efficient interference avoidance technique on unified MAC and PHY.

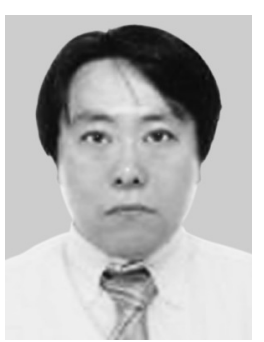

Takefumi Hiraguri received the M.E. and Ph.D. degrees from the University of Tsukuba, Ibaraki, Japan, in 1999 and 2008, respectively. In 1999, he joined the NTT Access Network Service Systems Laboratories, Nippon Telegraph and Telephone Corporation (NTT) in Japan. He has been engaged in research and development of high speed and high communication quality wireless LANs systems. He is now associate professor in Nippon Institute of Technology (NIT). He is a member of IEEE. 


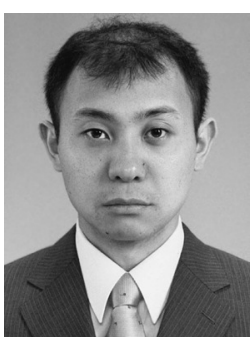

Masakatsu Ogawa received the B.E., M.E. and Ph.D. degrees from Sophia University, Tokyo, Japan, in 1998, 2000 and 2003, respectively. He was an adjunct member at National Institute of Informatics from 2003 to 2004, and a visiting researcher at Sophia University from 2003 to 2005. In 2004, he joined NTT Access Network Service Systems Laboratories, NTT Corporation. From 2004 to 2009, he was engaged in research and development of high speed wireless LANs. In 2009, he moved to the Technical Assistance and Support Center, NTT East Corporation. He received the IEICE Young Researcher's Award in 2007, and the IEICE Communications Society Distinguished Contributions Award in 2005, 2008 and 2009. He is a member of IEEE.

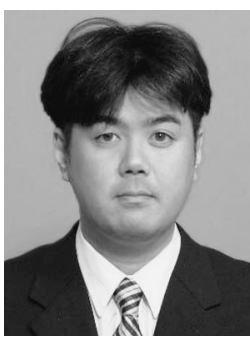

Kentaro Nishimori received the B.E., M.E. and Dr.Eng. degrees in electrical and computer engineering form Nagoya Institute of Technology, Nagoya, Japan in 1994, 1996 and 2002, respectively. In 1996, he joined the NTT Wireless Systems Laboratories, Nippon Telegraph and Telephone Corporation (NTT), in Japan. He was senior research engineer on NTT Network Innovation Laboratories. He is now associate professor in Niigata University. He was a visiting researcher at the Center for Teleinfrastructure (CTIF), Aalborg University, Aalborg, Denmark in 2006. He was an editor for the Transactions on Communications for the IEICE Communications Society and Assistant Secretary of Technical Committee on Antennas and Propagation of IEICE. He received the Young Engineers Award from the IEICE of Japan in 2001, Young Engineer Award from IEEE AP-S Japan Chapter in 2001, Best Paper Award of Software Radio Society in 2007 and Distinguished Service Award from the IEICE Communications Society in 2005 and 2008. His current research interest is Multi-user MIMO systems and cognitive radio systems. He is a member of IEEE.

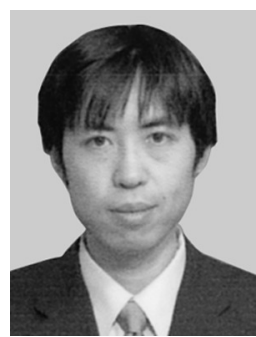

Naoki Honma received the B.E., M.E., and $\mathrm{Ph} . \mathrm{D}$. degrees in electrical engineering from Tohoku University, Sendai, Japan in 1996, 1998, and 2005, respectively. In 1998, he joined the NTT Radio Communication Systems Laboratories, Nippon Telegraph and Telephone Corporation (NTT), in Japan. He is now associate professor in Iwate University. He received the Young Engineers Award from the IEICE of Japan in 2003, the APMC Best Paper Award in 2003, and the Best Paper Award of IEICE Communication Society in 2006, respectively. His current research interest is planar antennas for high-speed wireless communication systems. He is a member of IEEE.

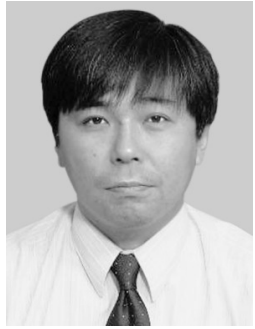

Tetsu Sakata received the B.E. and M.E. degrees from Kyoto Institute of Technology, Kyoto, Japan, in 1986 and 1988, respectively. He joined NTT Radio Communication Systems Laboratories in 1988. Since then he has been engaged in research and development of wireless LAN systems, digital modems, transmission power control system, and digital LSIs for satellite communications and personal communications. He is currently a Senior Research Engineer of Wireless Access Systems Project, NTT Access Network Service Systems Laboratories. He is a member of IEEE. 